Archives de sciences sociales des religions

131-132 | juillet - décembre 2005

Varia

\title{
Robert W. Hefner, Civil Islam. Muslims and Democratization in Indonesia
}

Avant-propos de James Piscator, Princeton (NJ), Princeton University Press, 2000, XXIV + 286 p.

\section{Hélène Bouvier}

\section{(2) OpenEdition}

\section{Journals}

Édition électronique

URL : http://journals.openedition.org/assr/3158

DOI : 10.4000/assr.3158

ISSN : $1777-5825$

Éditeur

Éditions de l'EHESS

Édition imprimée

Date de publication : 1 décembre 2005

Pagination : 215-311

ISBN : 2-7132-2045-9

ISSN : 0335-5985

Référence électronique

Hélène Bouvier, «Robert W. Hefner, Civil Islam. Muslims and Democratization in Indonesia », Archives de sciences sociales des religions [En ligne], 131-132 I juillet - décembre 2005, document 132-32, mis en ligne le 20 février 2006, consulté le 21 septembre 2020. URL : http://journals.openedition.org/assr/ 3158 ; DOI : https://doi.org/10.4000/assr.3158

Ce document a été généré automatiquement le 21 septembre 2020

(c) Archives de sciences sociales des religions 


\title{
Robert W. Hefner, Civil Islam. Muslims and Democratization in Indonesia
}

\author{
Avant-propos de James Piscator, Princeton (NJ), Princeton University \\ Press, 2000, XXIV + 286 p.
}

\section{Hélène Bouvier}

1 R. Hefner choisit « une perspective dialogique et transculturelle » pour « réfléchir sur la démocratie et la réforme religieuse dans une tradition non-occidentale » (p. XVII). Le livre se situe délibérément au cœur des débats qui ont parcouru les sciences sociales, en particulier l'anthropologie, tant sur la relativité culturelle que sur la place à donner à l'histoire. Le projet est de " proposer une anthropologie sociale de la démocratisation dans une société musulmane", et plus précisément d'expliquer l'émergence, dans l'islam indonésien des années 1980 et 1990, d'un mouvement "religieusement œcuménique et audacieusement réformiste " qui a aussi promu les droits des femmes, le dialogue inter-religieux et le combat pour la création d'un régime politique démocratique et pluraliste qui ne passe pas par un Etat islamique. L'auteur précise néanmoins que la «destinée du mouvement démocratique dépendra finalement non seulement du combat pour une société civile mais des efforts pour réformer l'Etat » (p. XVIII). Pour traiter cette question d'un islam civil, depuis haussée au rang des préoccupations d'actualité, R. Hefner saisit la complexité des forces en jeu et les multiples tournants dans les stratégies des différentes parties, même si le lecteur d'aujourd'hui est tenté d'appréhender les choses de manière plus réservée, connaissant les développements ultérieurs, alors inconnus de l'auteur.

2 Le premier chapitre "Democratization in an Age of Religious Revitalization " étudie deux éléments essentiels de la démonstration : d'une part, le pluralisme musulman au milieu de la diffusion transculturelle des idées démocratiques et de la réapparition en force des questions ethniques et religieuses dans les affaires publiques; d'autre part, le pluralisme culturel des musulmans d'Indonésie et leurs aménagements avec la culture locale (adat). Les deux chapitres suivants brossent l'arrière-plan des relations entre 
islam et politique, notamment l'importance du réseau rural et des écoles coraniques (pesantren), la construction du nationalisme et l'élaboration tumultueuse de la Constitution, dont le débat toujours actuel de savoir si l'Indonésie doit être un Etat non religieux ou un Etat islamique (appliquant notamment la shariah). Le quatrième chapitre « Ambivalent Alliances: Religion and Politics in the Early New Order » montre comment le régime a repoussé toute velléité politique des milieux musulmans tout en favorisant le développement de l'éducation et de la pratique religieuses, et comment, dans le paysage politico-religieux, se positionne le Nahdatul Ulama, association de musulmans traditionalistes fondée en 1926, clef de voûte de l'argumentaire de l'auteur sur l'islam démocratique.

3 Dans "The Modernist Travail ", l'auteur révèle comment deux grandes mouvances travaillent littéralement ce concept de manière contradictoire : d'un côté les « jeunes modernistes » qui considèrent que «la communauté musulmane devrait développer l'éducation et le bien-être social car, dans le long terme, les changements de société seront le meilleur moyen de progresser politiquement » (pp. 101-102) ; et de l'autre, un islam "anti-libéral», qui s'appuie sur le Conseil indonésien pour la Prédication islamique (Dewan Daqwah Islamiyah Indonesia, fondé en 1967), forge des liens avec des organisations et des donateurs d'Arabie Saoudite, du Koweit et du Pakistan (le lecteur d'aujourd'hui souhaiterait plus de détails sur ce point), et crée le journal «Media Daqwah ». Plus tard, en 1987, les plus conservateurs de ce Conseil fonderont le Comité indonésien pour la solidarité avec le monde islamique (Komite Indonesia untuk Solidaritas dengan Dunia Islam, KSDI) qui s'impliquera dans les turbulences violentes des années suivantes. La conquête de la classe moyenne musulmane devient alors l'enjeu principal, avec son entrée dans l'élite politique, le gouvernement, l'administration, les affaires. C'est le sujet du chapitre suivant «Islam Deferred: Regimist Islam and the Struggle for the Middle Class » qui dévoile comment, après avoir favorisé l'islam modéré à la fin des années 1980, le président Suharto joue la carte musulmane contre ceux qui, parmi les militaires et le mouvement pro-démocratie, ont commencé à contester sa loi. De fait, la création en 1990 de l'ICMI (Association des Intellectuels Indonésiens Musulmans), dénoncée par Abdurrahman Wahid, chef du NU, " comme étant une organisation "sectaire" qui est en train de reconfessionaliser la politique et la société » (p. 128), provoque rapidement des tensions profondes parmi les militaires, trouble les mouvements pro-démocrates multiconfessionnels et déclenche des débats enflammés au sein-même de la communauté musulmane. Finalement, en lui retirant sa faveur et son soutien, le régime choisit de cultiver les ultra-conservateurs, opportunément " régimistes », afin de casser la dynamique d'alliance naissante entre démocrates musulmans et non-musulmans.

4 Comme son titre l'annonce, le dernier chapitre « Uncivil State: Muslims and Violence in Soeharto's Fall» explore les batailles et retournements politiques des dernières années du régime, tout en posant la question de fond (toujours actuelle) : qu'advient-il des démocrates musulmans quand les élites dirigeantes choisissent les intérêts des réformateurs musulmans contre ceux des démocrates séculaires, et que l'islamisation est encouragée par un régime autoritaire ? Bien que le slogan « société civile » ait capté les esprits et "semblé ouvrir une voie plus aimable et douce vers la démocratie, qui éviterait la confrontation avec l'Etat [...], la réaction violente de Soeharto au mouvement pro-démocratie illustre la vérité générale selon laquelle, finalement, il ne peut y avoir de réelle société civile sans Etat civilisé » (p.189). Ainsi, R. Hefner démêle utilement pour le lecteur les stratégies « d'accommodation et de résistance » de Wahid, 
la reconfiguration politique des partis politiques, l'exploitation politique de la crise économique et financière de fin 1997, l'émergence d'une " opposition maintenant unie autour du NU de Wahid, du nationalisme de Megawati et des modernistes réformateurs de Rais» (p. 199), et l'alliance des musulmans régimistes du KISDI avec la faction militaire du général Prabowo. Leur campagne très préparée (voir le passage instructif sur le montage et l'utilisation du livret inflammatoire «The Conspiracy to Overthrow Soeharto", pp. 201-203) contre les "ennemis de l'Islam», chinois et chrétiens mais aussi musulmans modérés et ICMI, aboutira aux violences programmées de Java-est et Java-ouest en 1996, puis en mai 1998 à Jakarta. Après la démission forcée de Soeharto, entre la reprise de violences collectives à Jakarta, Java puis aux Moluques (et d'autres régions non mentionnées par l'auteur, comme Kalimantan ou Sulawesi) dans l'élan d'un agenda de division selon des lignes religieuses, politiques et ethniques, et les nouvelles violences autour de l'indépendance de Timor, Wahid est élu président, Megawati viceprésidente et Rais chef de l'Assemblée nationale. R. Hefner tempère néanmoins ce dénouement en ajoutant lucidement que les «avocats de la terreur étatique restent installés dans des segments de l'armée et de la bureaucratie. Le démantèlement de leur réseau de l'ombre - groupes d'auto-défense et gangsters - sera l'un des défis que l'Indonésie démocratique aura à affronter » (p. 213).

Dans sa conclusion, l'auteur exprime combien ce livre veut aussi témoigner du « rôle et du courage » des associations civiques musulmanes, qui déclarent "qu'il ne peut y avoir de contrainte dans la religion [qui] compte en fait la liberté, l'égalité et la justice parmi ses valeurs fondamentales ", ou encore reconnaissent combien il est important de « relier les commandements de Dieu à une interprétation œcuménique de l'histoire et de la culture indonésiennes » (p. 218). R. Hefner vérifie ainsi ses hypothèses initiales, opposées à celles de Huntington: "ces idéaux démocratiques ont une grande attractivité car ils répondent à des circonstances et à des besoins communs aux cultures modernes »; et plus loin, «le désir de démocratie et de moralité civile n'est pas circonscrit d'un point de vue civilisationnel. Cet espoir simple restera une force puissante en politique et en religion pour les années à venir » (p. 221).

Bien sûr, on est inévitablement tenté de se demander quelle serait l'analyse de R. Hefner pour la période suivante de 1999 à 2004, et ce qu'il aurait débusqué parmi les massacres et les conflits ethniques et religieux, les actes terroristes, les revendications indépendantistes, les premières élections présidentielles à suffrage direct et universel. Les forces en présence et " aux affaires », si bien mises en évidence dans cet ouvrage, révisent en ce moment-même un Code pénal vieillot, soumettant à discussion l'alourdissement des peines pour des articles touchant la liberté de la presse, la moralité ou les pratiques magiques. Les liens avec le monde musulman dans son ensemble ont pris une dimension complexe et incontournable, et ajoutent encore des acteurs sur l'échiquier. Cinq années plus tard, la réforme de l'Etat tant nécessaire n'a manifestement pas suivi le rythme de la société civile, faisant peser la même incertitude sur l'issue du processus de démocratisation indonésien à long terme. 